Nonlocal flow effects in bushfire spread rates

Dold, J.W. and Zinoviev, A. and Weber, R.O. 2006

MIMS EPrint: 2007.140

Manchester Institute for Mathematical Sciences

School of Mathematics

The University of Manchester

\footnotetext{
Reports available from: http://eprints.maths.manchester.ac.uk/

And by contacting: The MIMS Secretary

School of Mathematics

The University of Manchester

Manchester, M13 9PL, UK
} 


\title{
Nonlocal Flow Effects in Bushfire Spread Rates
}

\author{
John Dold \\ School of Mathematics, University of Manchester, Manchester M60 1QD, UK \\ $<$ john.dold@manchester.ac.uk> \\ Anna Zinoviev \\ School of Mathematics, University of Manchester, Manchester M60 1QD, UK \\ $<$ anna.zinoviev@manchester.ac.uk> \\ Rodney Weber \\ School of Physical, Environmental and Mathematical Sciences, UNSW at ADFA, \\ Canberra 2600,Australia <rodney.weber@unsw.adfa.edu.au> \\ and Bushfire CRC, Level 5, 340 Albert St., East Melbourne, Victoria 3002, Australia
}

\begin{abstract}
The entrainment of air into the bouyant flame and plume above any part of a fireline must affect the air flow at all other parts of the fireline. Thus the local wind actually felt at any one part of the fire is not necessarily the ambient wind; it is influenced, nonlocally, by all other parts of the fireline. By modelling this effect of bouyancy on the ambient air as a line of suction above the fireline, with a height and strength that varies with intensity of burning along the fireline, the changes in air flow can be calculated for any given shape and size of fireline. To illustrate the nature of the model, a fixed elliptical shape of fireline is examined within a steady ambient wind, as measured far from the fire. A reduction in head fire spread rate is predicted for finite widths of the fireline and, if the scale of the fireline increases, the spread rate approaches the limiting value of the potential spread rate. The model is therefore in qualitative agreement with the basic experimental findings of Cheney and Gould (1995).
\end{abstract}

Keywords: fire spread rate, modelling, air flow, entrainment

\section{Introduction}

Experimental observation of grassfires, as reported by Cheney and Gould (1995), has shown that the spread rate, found at the head of an elongated fire, is affected by the distance between the trailing flank fires, or the fire width. The rate of advancement of the head fire is found to increase as the fire width increases, only approaching the limiting potential spread rate, which is the spread rate of a long linear fire front (Cheney and Sullivan, 1997), at relatively large fire widths. The experiments carried out by Wotton et al (1999) on fires between cleared channels, which eliminated any trailing flank fires and generated head fires that propagated as more-or-less linear fire fronts, do not appear to show such a reduction in spread rate. This suggests that it is a property of the fire itself, as well as the shape of the fireline, that leads to a reduction in spread rate.

A physical explanation for the phenomenon lies in the effect that the net air indraft, needed to sustain one part of the fire, has on the flow of air at other parts of the fire. The strong buoyancy of the flame and the hot plume above the flame have the effect of drawing in ambient air from all directions, heating it and ejecting it vertically. The overall process is a nonlocal one because the spread rate at any one point on the fireline does not depend only on local conditions at that point; the air flow into any one part of the fire is influenced both by the prevailing wind and the burning and subsequent suction effect on the ambient 
air taking place everywhere else along the fire. Consequently, the rate of air flow into any one part of the fire depends on the overall shape of the rest of the fireline, as well as its scale and the intensity of burning. Also, because the overall effect of sucking decreases with distance, the process is weaker at larger scales, which is consistent with the observation that larger fire widths have less of an effect on spread rate.

It can be noted that the sucking caused by a straight line of head fire, with no flank fires, would only alter the flow at any part of the fire in a direction that is parallel to the fireline. This would not change the normal or perpendicular component of the air flow into the fireline at all and so should not be expected to alter the spread rate of the fire; this is consistent with the experimental findings of Wotton et al (1999). On the other hand, because trailing flank fires draw air away from a head fire in a direction that is not parallel to the line of the head fire, they must act to reduce the air flow into the head fire; backing parts of the fireline would contribute in the same way. The headfire should then spread more slowly than it would do if it experienced the full mean wind flow, uninfluenced by the sucking effect of other parts of the fireline.

This article introduces a relatively simple model formulation for the effect of sucking along a fireline. Key features are that: the rate of suction depends on the fireline intensity; its effect is taken to be focused along a path above the fireline, at a height that also depends on the fireline intensity; ambient air is drawn in from all directions above ground level into this path; the resulting air flow at any part of the fireline is then the integral effect of the mean wind and the sucking effect due to all other parts of the fireline; this, in turn, determines the spread rate and fireline intensity at any point on the fireline. Some effect of sucking was included in the cellular automaton model of Sullivan and Knight (2004) but only as a single point of sucking above the entire fireline. The still relatively simple but more realistic approach adopted here offers a useful generalisation that can be incorporated into fireline growth models, as for example reviewed by Pastor et al (2003). The sucking effect of the flame, as introduced in this model, provides a relatively simple representation of at least part of a physical process through which the overall shape and size of the fireline can affect its spread rate.

At this stage of development the model involves a number of parameters that are not yet known so that only its overall features are discussed and illustrated using an elliptical fireline of a fixed shape as an example. Calculations based on the model offer qualitative features that fit in with the overall experimental findings of Cheney and Gould (1995) and Wotton et al (1999). In particular, when flank fires exist, the head-fire spread rate is found to vary with the width of the elliptical fireline and to approach the potential spread rate at large widths. The model can clearly be extended to include the time dependent evolution of a fireline, for comparison with actual experimental fire evolutions and, eventually, for predictive purposes. Comparisons with experiment will help to calibrate the as yet unknown parameters within the model and work towards this end is currently in progress.

\section{Broad Features of the Fireline and the Flame}

It is informative firstly to examine some broad features of a fireline, evolving from a point ignition, assuming that there is a constant head-fire spread rate of $S_{\mathrm{h}}$ and a constant flankfire spread rate of $S_{\mathrm{f}}$, which can also be taken to represent the spread rate of the backing fire. Although, in reality, the head-fire spread rate is not constant, this approach is useful simply for establishing order of magnitude estimates of the relative extent and role of the different parts of the fire. 
The overall width of the head fire $L_{\mathrm{h}}$ (and also the backing fire) increases at a rate that is proportional to $2 S_{\mathrm{f}}$, as both flank fires move apart, so that $L_{\mathrm{h}} \approx 2 S_{\mathrm{f}} t$, where $t$ is the time from ignition. The length of the flank fires increases in proportional to $S_{\mathrm{h}}+S_{\mathrm{f}}$, as both the head and backing fires serve to extend the flank fires; that is $L_{\mathrm{f}} \approx\left(S_{\mathrm{h}}+S_{\mathrm{f}}\right) t$. If the same mass of vegetation is burnt per unit area in any part of the fire then the fireline intensities of the head and flank fires are simply proportional to the spread rate with some coefficient $Q$; that is $I_{\mathrm{h}} \approx Q S_{\mathrm{h}}$ and $I_{\mathrm{f}} \approx Q S_{\mathrm{f}}$.

The total power of the head fire can thus be estimated as $P_{\mathrm{h}} \approx Q S_{\mathrm{h}} L_{\mathrm{h}} \approx 2 Q S_{\mathrm{h}} S_{\mathrm{f}} t$ while $P_{\mathrm{f}} \approx Q S_{\mathrm{f}}\left(2 L_{\mathrm{f}}+L_{\mathrm{h}}\right) \approx Q S_{\mathrm{f}}\left(2 S_{\mathrm{h}}+4 S_{\mathrm{f}}\right) t \approx P_{\mathrm{h}}+2 P_{\mathrm{h}} S_{\mathrm{f}} / S_{\mathrm{h}}$ provides an estimate of the power of the combined flank and backing fires. While these arguments are not exact, particularly through assuming constant spread rates, they demonstrate that the combined flank and backing fires release a similar amount of energy per unit time $P_{\mathrm{f}}$ to the head fire $P_{\mathrm{h}}$, or possibly more energy if the head-fire spread rate is not very much larger than that of the flank and backing fires. Hence, even though the head fire may dominate the overall advancement of the fire, the flank and backing fires can be equally as significant in terms of the energy they release and all related effects such as their contribution to buoyancy and subsequent convection in the atmosphere.

Secondly, the complete overall burning of carbohydrate (in almost any form) can be represented approximately as $n \mathrm{CH}_{2} \mathrm{O}+n \mathrm{O}_{2} \rightarrow n \mathrm{CO}_{2}+n \mathrm{H}_{2} \mathrm{O}$. For one molecular mass (30) of $\mathrm{CH}_{2} \mathrm{O}$ the molecular mass (32) of an oxygen molecule $\mathrm{O}_{2}$ is consumed from the air. Thus a similar mass of oxygen is consumed to the dry mass of vegetation that is burned. Since oxygen constitutes $21 \%$ of air (by volume) and 32 grams of oxygen occupy 22.4 litres at ambient atmospheric conditions, burning one kilogram of dry vegetation requires about 3.5 cubic metres of air. If this were the only means by which air was drawn into the fire, then a fireline spreading at 1 metre per second and burning $1 \mathrm{~kg}$ of dry vegetation per square metre (a dry fuel load of 10 tonnes per hectare) would draw in about 3.5 cubic metres of ambient air per metre of fireline per second, simply in order to supply enough oxygen to sustain the combustion.

However, this is likely to be an underestimate of the rate of entrainment of air. The products of the burning are strongly buoyant hot gases, having a density that is about one seventh that of air which must therefore be accelerated vertically at almost the acceleration of gravity, entraining additional air as they rise. Once the chemistry is over, so that the flame stops releasing heat, the entrainment of air can cool the gases, which continue to rise as a buoyant plume. The overall effect of the burning in any part of a fireline thus involves the inflow of ambient air both to provide oxygen to sustain the chemistry and to be entrained into the hot rising gases. Without entering into any more details of the process, it is clear that the rate of entrainment $\pi \gamma$ of ambient air, per unit length of the fireline, can be expected to be approximately proportional to the fireline intensity $I$, having the form $\pi \gamma=c I$ for a constant of proportionality $c$.

The hot gases produced by the combustion and by the entrainment and heating of cooler gases are preferentially ejected vertically, due to the strong buoyancy. On the other hand, the ambient air that is sucked into the fire is drawn from all directions above ground level (apart from the relatively narrow flame and plume). If the entrainment rate $\pi \gamma \mathrm{d} s$ above a portion of the fireline of arclength $\mathrm{d} s$ is focused at a point in open space, then the resulting velocity $\mathrm{d} v$ of air flow towards the point, at a distance $r$ from the point, is simply $\mathrm{d} v=\gamma \mathrm{d} s /\left(4 r^{2}\right)$. This inverse square law ensures that a volume $\pi \gamma \mathrm{d} s$ of air enters any sphere of radius $r$, centred on the point, per unit time. If the point where the sucking is 


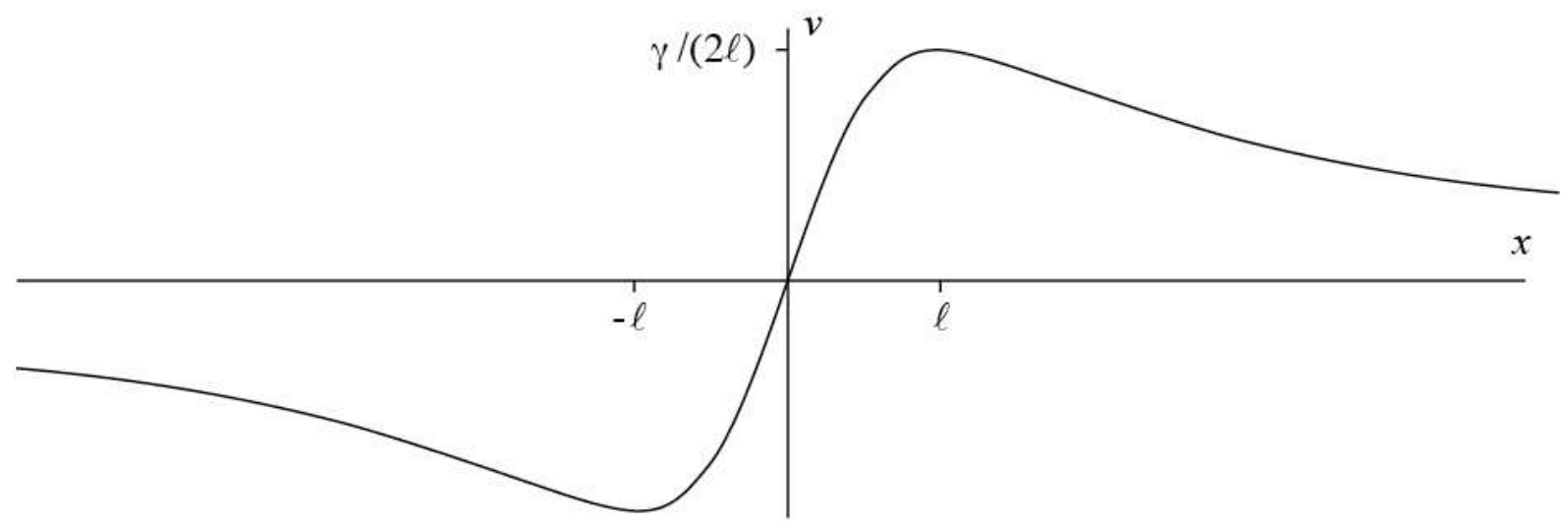

Figure 1: The speed $v$ of the induced flow of air, near ground level, into an idealised straight fireline, induced by a sucking of strength $\pi \gamma$ cubic metres per second per metre of fireline, effectively focused at a height $\ell$ metres above an impermeable ground level. The induced flow is zero at the fireline, has a maximum value of $\gamma /(2 \ell)$ metres per second at a distance $x=\ell$ and decreases as the distance $x$ from the fireline increases further.

focused is at a height $\ell$ above horizontal impermeable ground, then the flow at any point can be calculated as the vector sum of the flows due to two points of sucking, each of strength $\pi \gamma \mathrm{d} s$, with one located at the height $\ell$ above ground and the other being a virtual sink at the reflected position of the actual sink, namely at the depth $\ell$ below ground. The sink and its reflection simply ensure that no air flows through the ground while giving the correct flow velocity above ground for an ideal or inviscid fluid. In the context of a bushfire, the flow velocity should only be incorrect within a relatively thin viscous boundary layer close to the surface of the ground.

The flow field in the idealised case of an infinite straight line of fire, can then be calculated exactly. The horizontal component of the induced flow in a direction normal to the fireline, measured close to ground level but above any viscous boundary layer, is found to be given by $\gamma x /\left(\ell^{2}+x^{2}\right)$, flowing towards the fireline, where $x$ is the distance from the fireline. This flow speed has a maximum value of $\gamma /(2 \ell)$ at the distance $\ell$ from the fireline, as sketched in Figure 1. As an illustration, for a fire that entrains 3.5 cubic metres of ambient air per metre of fireline per second, with $\ell \approx 1 / 2$ metre, the maximum induced flow speed would be approximately $3.5 / \pi \approx 1.1$ metres per second, found at a distance of $1 / 2$ metre on either side of the fireline. The induced flow speed falls away at greater distances from the fireline and is zero at the fireline itself (where $x=0$ ).

In reality, the entrainment above any part of the fireline is not focused at a point although it is convenient to model it this way. Rather, the effect of the sucking on the ambient air is distributed in some way through the flame and the plume above the fireline. While it may be possible to generalise the model, treating the sucking as though it were focused at a point of height $\ell$ above any one part of the fireline of length $\mathrm{d} s$ introduces an important feature that is missing from other fireline growth models to date; see for example the review of Pastor et al (2003). In principle, measurements of induced flow around straight lines of fire, as just described, could be used to offer experimental fits for the rate of entrainment $\pi \gamma$ and an effective entrainment height $\ell$. 


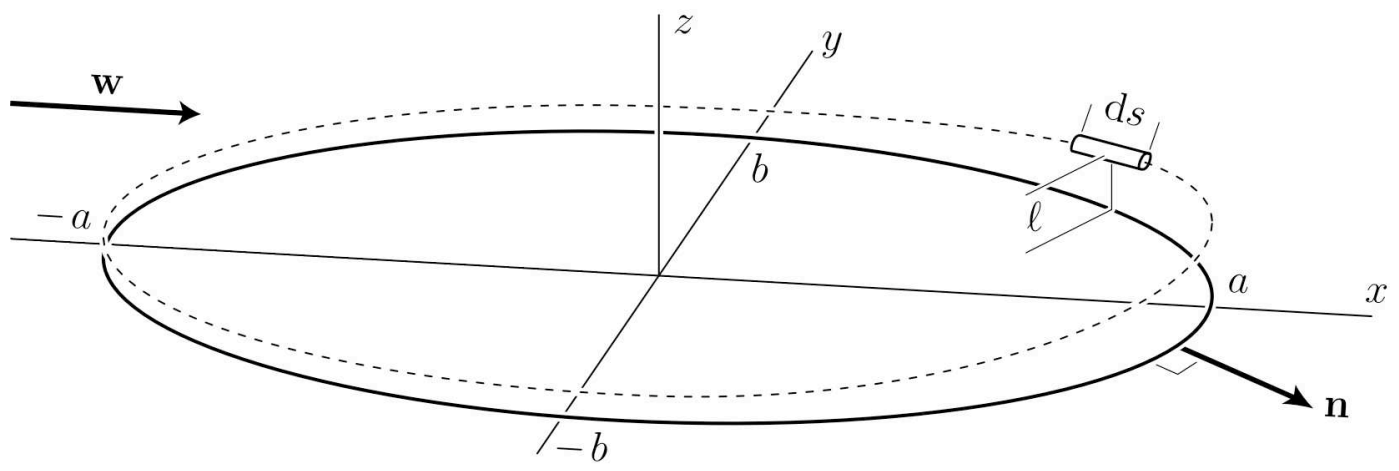

Figure 2: Illustration of an elliptical fireline showing a normal vector $\mathbf{n}$, the wind $\mathbf{w}$ and a small element of length $\mathrm{d} s$ at a height $\ell$ above horizontal where the sucking effect of the fire is modelled.

In summary, simple arguments on the relative spread rates of head fires and flank and backing fires suggest that the total power of flank and backing fires is normally at least as great as the power of the more rapidly spreading head fire, unless for some reason the flank fires are extinguished as in the experiments of Wotton et al (1999). The overall rates of sucking or entrainment caused by these two kinds of fire can therefore be estimated to be similar. The flank fires should therefore be able to diminish the actual flow of air into the head fire leading to a reduction in head-fire spread rate. Relatively straightforward arguments suggest that the sucking may be characterised in terms of an intensity of sucking $\gamma$ and an effective height of sucking $\ell$ both of which should vary along the fireline as the intensity of the fire $I$ and the spread rate $S$ vary. For a given distribution of $\gamma$ and $\ell$ along a given fireline, an inverse square law for the flow induced by the sucking offers a way of calculating the integrated effect of the sucking on the flow at any point on the fireline, providing a correction to the wind speed that is actually experienced at different points along the fireline. A spread rate based on this wind speed will generally be different from the potential spread rate based on the ambient wind speed measured far from the fire.

\section{Model and its Application to an Elliptical Fireline}

An outwardly propagating fireline can be represented by a closed path on a twodimensional flat horizontal terrain, mapped out using $x$ and $y$ coordinates. For example, an elliptical fireline, following the path $x^{2} / a^{2}+y^{2} / b^{2}=1$, is illustrated in Figure 2, in which $z$ measures vertical height. To make matters simpler, a horizontal mean wind of speed $\mathbf{w}$ can be taken to flow in the $x$ direction and to be independent of height. Using $\mathbf{i}, \mathbf{j}$ and $\mathbf{k}$ to represent unit vectors in the $x, y$ and $z$ directions, the wind is then the vector $\mathrm{w}=w \mathbf{i}$. If this was the only air flow that the fire experienced, then the normal component of the flow into any part of the fire would be $u_{\mathrm{n}}=\mathbf{w} \cdot \mathbf{n}$ where $\mathbf{n}$ is the unit normal to the fireline. Taking the spread rate $S$ to depend only on the normal flow $u_{\mathrm{n}}$, this would ensure that the head of the fire (where $\mathbf{w}$ and $\mathbf{n}$ have the same direction) would propagate at the full potential spread rate regardless of the shape and extent of the fireline.

However, the generation of hot gases and their strong bouyancy alter the local air flow. The rising of these hot gases must be balanced by the drawing of fresh ambient air 

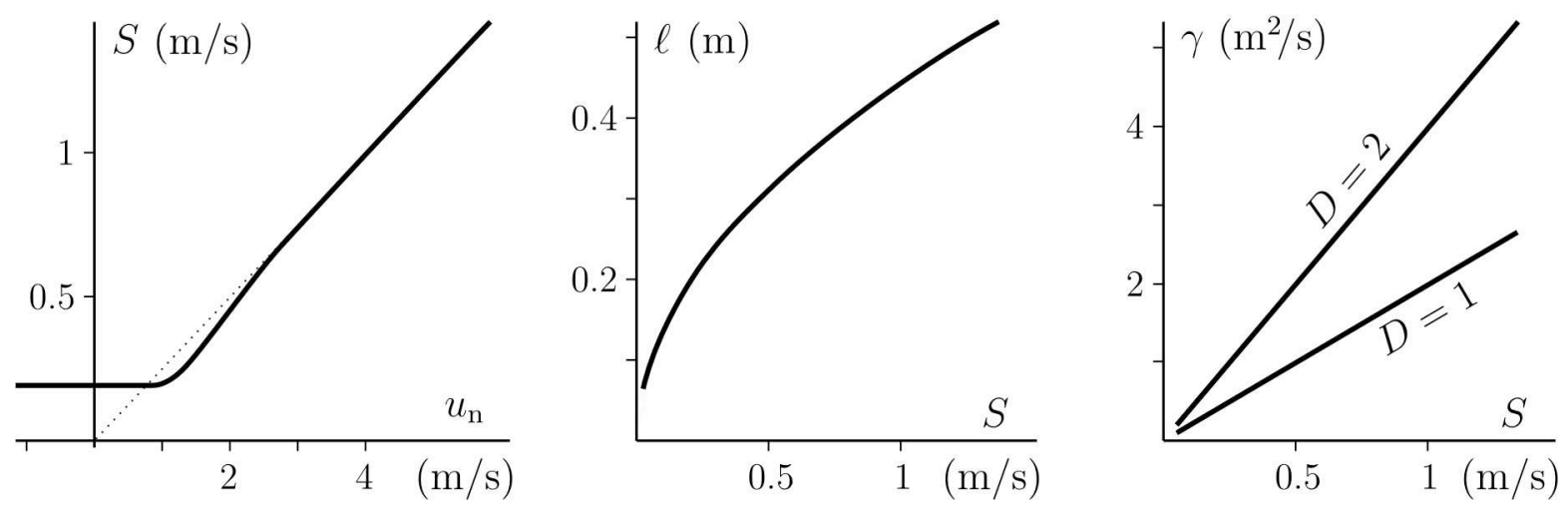

Figure 3: Left: the spread rate $S$ as a function of the normal air flow $u_{\mathrm{n}}$. Centre: the height $\ell$ as a function of the spread rate $S$. Right: two models for the intensity of sucking $\gamma$ as functions of the spread rate $S$ (labelled with $D=1$ and $D=2$ ).

towards the fire, where the air oxidizes the pyrolysis products of the heated vegetation and maintains the generation of hot combustion products. The fire is, effectively, a sink of ambient air, the strength of which can be represented by $\pi \gamma \mathrm{m}^{3} / \mathrm{s}$ (per metre of the fireline). At different parts of the fire, the "sucking strength" $\gamma$ would be different, depending on the spread rate $S$ and the energy content of the vegetation, per square metre, that is released as the fire front progresses (that is, the fire intensity $I$ ).

To model this in a simple way, the sucking can be taken to be concentrated at a height $\ell$ above the fireline. Again, $\ell$ might change with fire intensity. This means that, above any small arclength $\mathrm{d} s$ of the fireline at a position $(x, y)$, there would be a sink of strength $\pi \gamma \mathrm{d} s \mathrm{~m}^{3} / \mathrm{s}$, located at the position $x \mathbf{i}+y \mathbf{j}+\ell \mathbf{k}$, as illustrated in Figure 2. This sink draws in air from the entire region above ground level and ejects it into the bouyant plume. Outside the relatively narrow plume, the velocity of the flow that the sink generates at any point in an open environment is directed towards the sink at a speed $\gamma \mathrm{d} s /\left(4 r^{2}\right) \mathrm{m} / \mathrm{s}$, where $r$ is the distance from the sink. Treating the air as an ideal fluid, the flow $\mathbf{v}$ at any part of the fireline, that is induced by this sucking from all other parts of the fireline, can then be calculated by integrating over all of the contributions from the entire fireline, taking into account the fact that air does not flow through the ground by including a virtual path of reflected sinks at a depth $\ell$ below ground level.

The normal component of the air flow into the fire $u_{\mathrm{n}}$ can then be calculated using both the wind $\mathbf{w}$ and the fire induced flow $\mathbf{v}$, giving $u_{\mathrm{n}}=(\mathbf{w}+\mathbf{v}) \cdot \mathbf{n}$. This normal air flow does depend on the shape and size of the fire and would not generally allow a head fire to propagate at the potential spread rate, unless the fireline were indeed a perfectly straight line. In order to examine these effects, calculations were carried out for specified elliptical firelines of aspect ratio 2 (having $a=2 b$ and fire width $2 b$ ) for various values of the wind speed $\mathbf{w}$. Figure 3 shows the dependence on $u_{\mathrm{n}}$ of the spread rate $S$, that was used in these calculations, as well as the dependence on $S$, used to model the sucking intensity $\gamma$ and sucking height $\ell$. Two sets of calculations were carried out, one for each of the two straight line models for $\gamma$ shown in Figure 3; the slope of each line is proportional to the parameter $D$.

The resulting spread rates of the head fire for $D=1$ and $D=2$ are shown in Figures 4 and 5. These demonstrate a dependence of the head fire spread rate on fire width. In all 


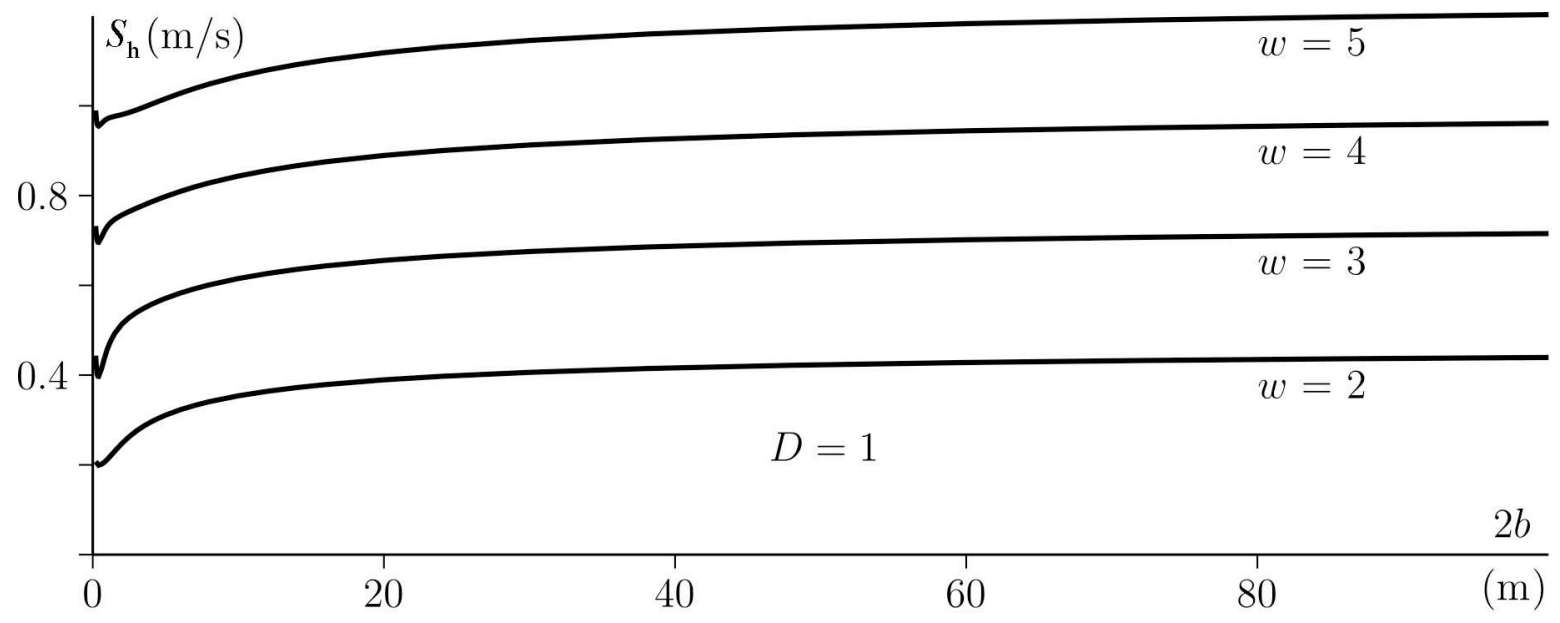

Figure 4: Head fire spread rate $S_{\mathrm{h}}$ as a function of the fire width $2 b$ for an elliptical fireline of aspect ratio 2 in the ambient wind speeds $w=2,3,4$ and $5 \mathrm{~m} / \mathrm{s}$, as calculated in the case where the sucking intensity is modelled using the value $D=1$.

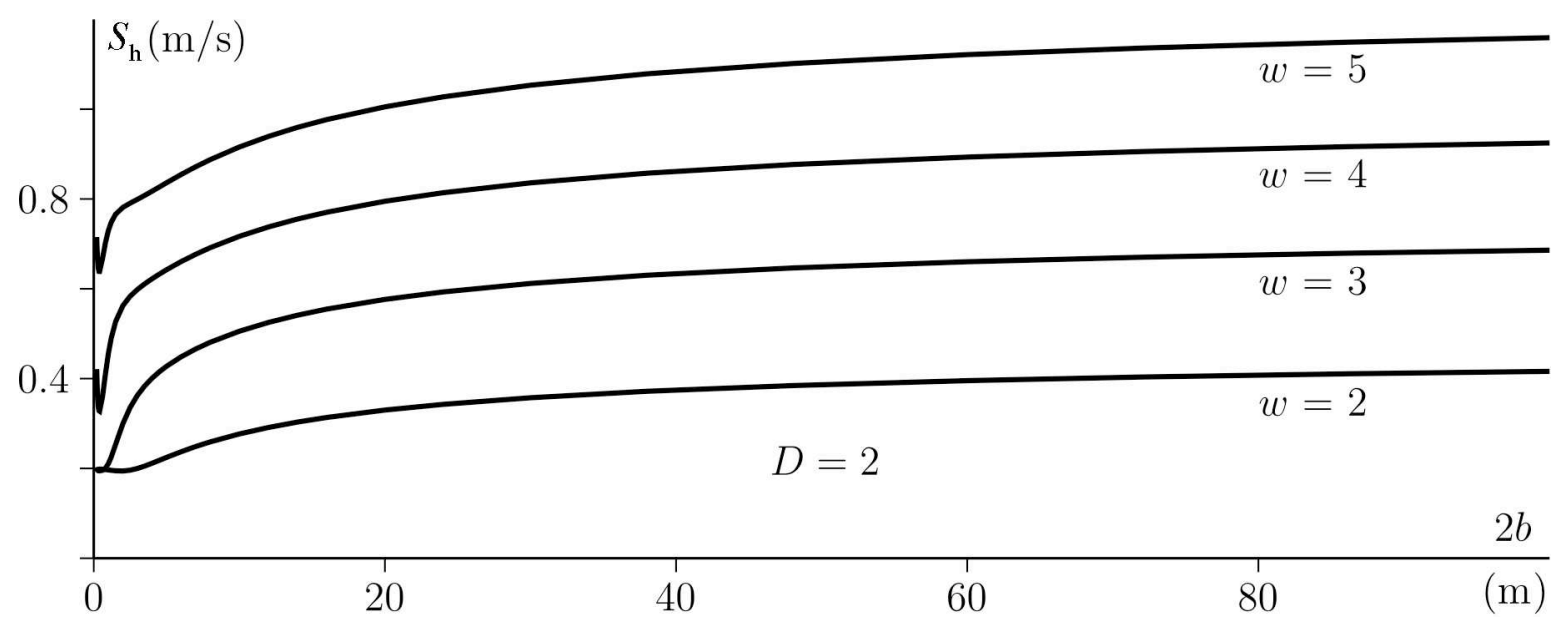

Figure 5: Head fire spread rate $S_{\mathrm{h}}$ as a function of the fire width $2 b$ for an elliptical fireline of aspect ratio 2 in the ambient wind speeds $w=2,3,4$ and $5 \mathrm{~m} / \mathrm{s}$, as calculated in the case where the sucking intensity is modelled using the value $D=2$.

cases the calculated spread rate increases as fire width increases, starting from its value at a small fire width of the order of $\ell$. It only approaches the potential spread rate as the fire width becomes relatively large. As should be expected, the effect is more pronounced in the case $D=2$ which involves a stronger level of sucking.

\section{Conclusions}

Of course, these calculations serve only to illustrate the essential nature of the model. A real developing fireline is unlikely to be an ellipse with an aspect ratio of exactly two and more complete time-evolving calculations are needed for more precise comparison with 
experimental records of bushfires. Nevertheless the calculated dependence of head-fire spread rate on fire width is qualitatively correct, which shows that the modelling procedure outlined here captures at least some aspects of the nonlocal interaction that strong buoyancy must cause between different parts of a fireline.

The calculations are based on the notion that the sucking of ambient air can be modelled as occurring with an intensity measured by $\pi \gamma$ per unit length of fireline at a height $\ell$ above the fireline (with impermeable ground taken into account using a reflected line of sucking). Both $\gamma$ and $\ell$ vary along the fireline, depending on the local fireline intensity $I$ and the local spread rate $S$, which both depend on the local normal flow rate $u_{\mathrm{n}}$ into the fireline. In turn, $u_{\mathrm{n}}$ depends on $\gamma$ and $\ell$ through the flow that is induced by the sucking, so that the problem is mathematically closed for a given fireline and for given dependences of $\gamma$ and $\ell$ on the spread rate $S$ and the dependence of $S$ on $u_{\mathrm{n}}$. For the solutions presented here, these dependences are all sketched in Figure 3. A simple extension of the model is to use the spread rate $S$ that can be calculated in this way, at any point on the fireline, to calculate how a fireline evolves over time.

The main challenge for further development is to identify relationships between $\gamma$, $\ell, S$ and $u_{\mathrm{n}}$ that fit in with experimental records of evolving firelines. There is clearly a fair degree of flexibility in modelling these relationships so that suitably chosen models should be able to provide a reasonably good reproduction of the experimental records. Work along these lines is currently in progress.

Acknowledgements: The authors are grateful for the financial support of EPSRC, Leverhulme Trust, MIMS, ADFA and Bushfire CRC.

\section{References}

Cheney, N.P. and Gould, J.S., 1995. Fire growth in grassland fuels. International Journal of Wildland Fire 5, 237-247.

Cheney, Phil and Sullivan, Andrew, 1997. Grassfires. CSIRO Publishing, Melbourne.

Pastor, E., Zárate, L., Planas, E. and Arnaldos, J., 2003. Mathematical Models and Calculation Systems for the Study of Wildland Fire Behaviour. Progress in Energy and Combustion Science 29, 139-153.

Sullivan, A.L. and Knight, I.K. (2004) A hybrid cellular automata/semi-physical model of fire growth. Proceedings of the 7th Asia-Pacific Conference on Complex Systems, Cairns, Australia.

Wotton, B.M., McAlpine, R.S. and Hobbs, M.W., 1999. The effect of fire front width on surface fire behaviour. International Journal of Wildland Fire 9, 247-253. 